\title{
Influence of age on bronchoscopic findings in healthy beagle dogs
}

\author{
E. Mercier ${ }^{\mathrm{a}, *}$, M. Bolognin ${ }^{\mathrm{a}}$, A.C. Hoffmann ${ }^{\mathrm{a}}$, C. Tual ${ }^{\mathrm{a}}$, M.J. Day ${ }^{\mathrm{b}}$, C. Clercx ${ }^{\mathrm{a}}$ \\ ${ }^{a}$ Department of Veterinary Clinical Sciences, Faculty of Veterinary Medicine, University of Liège, Boulevard de Colonster 20(B44), 4000 Liège, Belgium \\ ${ }^{\mathrm{b}}$ Division of Veterinary Pathology, Infection and Immunity, School of Clinical Veterinary Science, University of Bristol, Langford BS40 5DU, United Kingdom
}

\section{A R T I C L E I N F O}

Article history:

Accepted 8 December 2009

\section{Keywords:}

Bronchoscopy

Bronchoalveolar lavage

Canine

Age

\begin{abstract}
A B S T R A C T
The aims of this study were to evaluate the effect of age on bronchoscopic features and bronchoalveolar lavage fluid (BALF) cellularity in dogs. Thirty healthy beagle dogs from three age groups were included: young dogs ( 10 months to 4.5 years of age; $n=8$ ), middle-aged dogs $(5-8$ years old; $n=13$ ) and older dogs (>8 years; $n=9$ ). Haematology, thoracic radiography, bronchoscopy and bronchoalveolar lavage were performed; bronchoscopic findings were scored and BALF total and differential cell counts were determined. The total bronchoscopic score was higher in older dogs; these dogs had more irregular bronchial mucosa, more prominent mucosal vessels and bronchiectasis. Younger dogs had a higher percentage of neutrophils in BALF compared with middle-aged and old dogs and a higher percentage of lymphocytes in BALF compared with middle-aged dogs. The results show that age has an effect on bronchoscopic features of airways and the composition of BALF in the dog.
\end{abstract}

(c) 2009 Elsevier Ltd. All rights reserved.

\section{Introduction}

Bronchoscopy is an important adjunctive procedure in evaluation of canine respiratory disease. The technique permits visual assessment of the upper and lower respiratory mucosa, evaluation of dynamic airway changes and collection of deep respiratory samples for cytology, histology and culture (McKiernan, 2003). A good understanding of the normal structure of the bronchial tree is required if the clinician is to recognise subtle abnormalities and diseases (Kuehn and Hess, 2004). Scoring systems based on predefined macroscopic findings are used to improve objectivity in assessment of the type and severity of bronchial lesions (Clercx et al., 2000).

In healthy dogs, thoracic radiographic findings (Reif and Rhodes, 1966) and tracheobronchial mucociliary clearance (Whaley et al., 1987) are known to be age dependent. One study of 18 Beagle dogs of various ages reported no age-related changes in total and differential cell counts (TCC and DCC) in bronchoalveolar lavage fluid (BALF) (Mayer et al., 1990). There are no other published data concerning age-related differences in bronchoscopic findings in this species. The aim of the present study was to determine whether there was an effect of age on bronchoscopic appearance of airways and cellularity of BALF in the dog.

\footnotetext{
* Corresponding author. Tel.: +32 4 3664200; fax: +32 43664241.

E-mail address: emercier@ulg.ac.be (E. Mercier).
}

\section{Materials and methods}

Animals and inclusion criteria

This cross-sectional study was approved by the Animal Ethical Committee of the University of Liège (Approval Nos. 556, 649 and 663) and included 30 healthy experimental Beagle dogs from a colony at the Liège University Veterinary Small Animal Teaching Hospital. Dogs were assigned to one of three age groups: Group I: young dogs, 10 months to 4.5 years of age (mean \pm standard error of the mean, SEM, $2.1 \pm 0.6$ years; $n=8$ ); Group II: middle-aged dogs, $5-8$ years of age (mean \pm SEM, $7.3 \pm 0.1$ years; $n=13$ ); Group III: old dogs, $>8$ years (mean $\pm \mathrm{SEM}$, $12.2 \pm 0.3$ years; $n=9$ ).

All animals were bred and born in our facilities. The dogs spent their entire life in the same environmental conditions (wood shaving litter), with identical feeding, vaccinations and handling procedures, and were housed in groups of two or three. Some dogs were siblings. Anthelminthic treatments were administered to all dogs every 6 months and within 3 months before the onset of the study.

All dogs were considered to be clinically normal and had no clinical signs consistent with respiratory disease or any other systemic disease during the 3 month period preceding sampling. No abnormalities were detected on physical examination, haematology, serum biochemistry, thoracic radiography and BALF analysis. A nasal swab from one dog in each group was negative for canine parainfluenza virus type 2 by PCR. No significant pathogens were detected on bacterial culture of a nasal swab from one dog in each group. No serological testing for canine respiratory viruses was undertaken.

\section{Thoracic radiographs}

Right lateral and ventrodorsal thoracic radiographs were taken before bronchoscopic examination in each dog. A scoring system was used to evaluate the bronchial, interstitial and alveolar patterns (Foster et al., 2004). The bronchial pattern was scored as: 0 , absence of changes; 1 , mild (primary bronchi visible); 2 , moderate (secondary bronchi visible); 3 , severe (tertiary bronchi visible). The interstitial pattern was scored as: 0 , no changes; 1 , mild (mild interstitial framework visible); 2 , moderate (interstitial framework distinguishable from a bronchial pattern); 3 , severe (clearly apparent interstitial pattern). The alveolar pattern was scored as: 0 , 
no changes; 1 , mild (mild, localised alveolar pattern); 2 , moderate (moderate, bilateral alveolar pattern); 3, severe (clearly apparent bilateral alveolar pattern). Radiographs were reviewed blindly and radiographic scores were assigned by one evaluator $(\mathrm{ACH})$. The total score ranged from 0 to 9 .

\section{Bronchoscopy}

All dogs were premedicated by intravenous administration of medetomidine $(100 \mu \mathrm{g} / \mathrm{kg}$; Domitor, Pfizer Animal Health). A 5-min preoxygenation period was used and oxygen saturation was controlled during the procedure. Anaesthesia was induced (1-2 mg/kg) and maintained $(0.1-0.2 \mathrm{mg} / \mathrm{kg}$ ) by intravenous administration of propofol (Diprivan, Astra Zeneca). During recovery, dogs received supplementary oxygen as needed and anaesthesia was reversed by intramuscular administration of atipamezole $(50 \mu \mathrm{g} / \mathrm{kg}$; Antisedan, Pfizer Animal Health).

Bronchoscopy was performed using a paediatric $4.8 \mathrm{~mm}$ diameter video-endoscope (Fujinon EB-4105, ONYS). A scoring system was used to define the gross bronchoscopic features: bronchial mucosal surface $(0$, smooth; 1 , mild irregularity; 2 , markedly irregular), bronchial mucosal colour (0, pale; 1 , erythematous; 2 , both white and red areas), bronchiectasis ( 0 , no airway enlargement; 1 , single dilated bronchus; 2 , more than one dilated bronchus), prominence of vessels ( 0 , no visible vessels; 1 , visible vessels, blue; 2 , large, distended blue vessels; 3 , very large, protruding, tortuous vessels; these scores were increased by 1 if pulsation of the vessels was seen); decreased bronchial diameter during expiration $(0$, no decrease; 1 , mild collapse; 2 , moderate collapse; 3 , severe collapse, i.e. bronchomalacia). Video footage was reviewed blindly and bronchoscopic scores were assigned together by two evaluators (CC and EM). The maximum total bronchoscopic score was 13.

\section{Bronchoalveolar lavage}

BAL was performed as previously described (Clercx et al., 2000). Three aliquots of $15 \mathrm{~mL}$ sterile saline solution $(0.9 \%)$ at $37{ }^{\circ} \mathrm{C}$ were used. The first two aliquots were instilled successively, whilst the endoscope was wedged into a bronchus inside the left diaphragmatic lobe. The third aliquot was instilled into a bronchus inside the right diaphragmatic lobe. Each aliquot was instilled through a three-way stopcock into the biopsy channel by syringe, then withdrawn after $3 \mathrm{~s}$ by low-power pump aspiration and collected into a single sterile container. The volume of the pooled recovered fluid was measured.

Two millilitres of the retrieved fluid were placed into a tube containing ethylenediamine tetraacetic acid for determination of TCC by an automated haemocytometer (Coulter Gen.S). A second aliquot was stored at $4{ }^{\circ} \mathrm{C}$, then centrifuged within $6 \mathrm{~h}$ at room temperature for $4 \mathrm{~min}$ using a Shandon Cytospin 4 (Thermo Electron). Cytospin slides were stained by the May-Grünwald-Giemsa technique and examined microscopically. Differential cell counts were determined by counting 300 cells at high power magnification (63× objective). Epithelial cells and isolated nuclei were not counted.

\section{Statistical analysis}

Radiographic and bronchoscopic scores and numbers and percentages of blood and BALF leucocytes were tested for normality. Differences between the three groups of dogs were assessed using either one way analysis of variance (ANOVA) or the Kruskall Wallis test. Coefficients of linear regression $(r)$ and associated probabilities $(P)$ were determined in order to examine the relationship between radiographic scores, bronchoscopic scores and total cell count. All values are reported as means \pm SEM. A value of $P<0.05$ was considered to be statistically significant.

\section{Results}

Details of experimental dogs are shown in Table 1. Body weights and haematological variables were comparable in all groups.

\section{Radiographic scores}

There were no significant differences in total, bronchial and interstitial radiographic scores between groups (Table 1). No dog had a score of 0 and alveolar lesions were not observed.

\section{Bronchoscopic scores}

Total bronchoscopic score was significantly higher in older dogs than middle-aged dogs and younger dogs $(P<0.001)$ (Fig. 1$)$. In older dogs, the bronchial mucosa was significantly more irregular, there were more prominent vessels and bronchiectasis was observed (Fig. 2).

\section{Bronchoalveolar lavage fluid analysis}

Mean recovery rate of instilled fluid was $65 \pm 9 \%$. Mean TCC in BALF (cells $/ \mu \mathrm{L}$ ) was similar in the three groups. Younger dogs had higher percentages of neutrophils than middle-aged and older dogs and higher percentages of lymphocytes compared with middle-aged dogs (Table 1 ).

Table 1

Summary of signalment, laboratory data and radiographic scores.

\begin{tabular}{|c|c|c|c|c|c|}
\hline & & Group I $(n=8)$ & Group II $(n=13)$ & Group III $(n=9)$ & $P$ \\
\hline Age (years) & & $2.1 \pm 0.6$ & $7.3 \pm 0.1^{\mathrm{a}}$ & $12.2 \pm 0.3^{b}$ & $<0.05$ \\
\hline Age range (years) & & $0.8-4.5$ & $6-7.8$ & $11-14$ & NS \\
\hline Body weight (kg) & & $15.7 \pm 1.5$ & $16.2 \pm 0.8$ & $17.2 \pm 0.3$ & NS \\
\hline Males (M); females (F) & & $5 \mathrm{M} ; 3 \mathrm{~F}$ & $4 \mathrm{M} ; 9 \mathrm{~F}$ & $6 \mathrm{M} ; 3 \mathrm{~F}$ & \\
\hline \multirow[t]{5}{*}{ Blood leucocytes (number/ $\mu \mathrm{L}$ ) } & Total & $10100.0 \pm 848.0$ & $9508.3 \pm 819.8$ & $8025.0 \pm 693.3$ & NS \\
\hline & Neutrophils & $6820.2 \pm 597.1$ & $6768.7 \pm 571.3$ & $5645.2 \pm 751.8$ & NS \\
\hline & Eosinophils & $678.0 \pm 347.2$ & $412.8 \pm 88.7$ & $570.0 \pm 146.3$ & NS \\
\hline & Lymphocytes & $2129.6 \pm 281.7$ & $1811.4 \pm 225.7$ & $1271.2 \pm 202.7$ & NS \\
\hline & Monocytes & $472.1 \pm 99.1$ & $515.4 \pm 112.5$ & $538.5 \pm 86.2$ & NS \\
\hline \multirow[t]{4}{*}{ Radiographic score } & Total & $3.1 \pm 0.3$ & $3.7 \pm 0.2$ & $3.7 \pm 0.3$ & NS \\
\hline & Interstitial & $1.4 \pm 0.1$ & $1.9 \pm 0.2$ & $1.7 \pm 0.1$ & NS \\
\hline & Bronchial & $1.7 \pm 0.2$ & $1.8 \pm 0.2$ & $2.1 \pm 0.2$ & NS \\
\hline & Alveolar & $0 \pm 0$ & $0 \pm 0$ & $0 \pm 0$ & NS \\
\hline BALF TCC (number $/ \mathrm{mm}^{3}$ ) & & $377.5 \pm 88.8$ & $287.7 \pm 48.8$ & $501.3 \pm 68.6$ & NS \\
\hline \multirow[t]{4}{*}{ BALF DCC (\%) } & Macrophages & $71.41 \pm 5.55$ & $88.61 \pm 1.29$ & $79.77 \pm 5.44$ & NS \\
\hline & Neutrophils & $19.36 \pm 3.78$ & $9.53 \pm 1.07^{\mathrm{a}}$ & $9.46 \pm 2.03^{b}$ & 0.025 \\
\hline & Lymphocytes & $5.88 \pm 1.39$ & $1.11 \pm 0.30^{\mathrm{a}}$ & $5.09 \pm 1.96$ & 0.012 \\
\hline & Eosinophils & $3.38 \pm 1.97$ & $0.76 \pm 0.25$ & $5.70 \pm 3.37$ & NS \\
\hline
\end{tabular}

Mean \pm SEM is presented for each parameter.

NS, non-significant; BALF, bronchoalveolar lavage fluid; TCC, total cell count; DCC, differential cell count.

a Significant difference between group I and group II.

b Significant difference between group I and group III. 


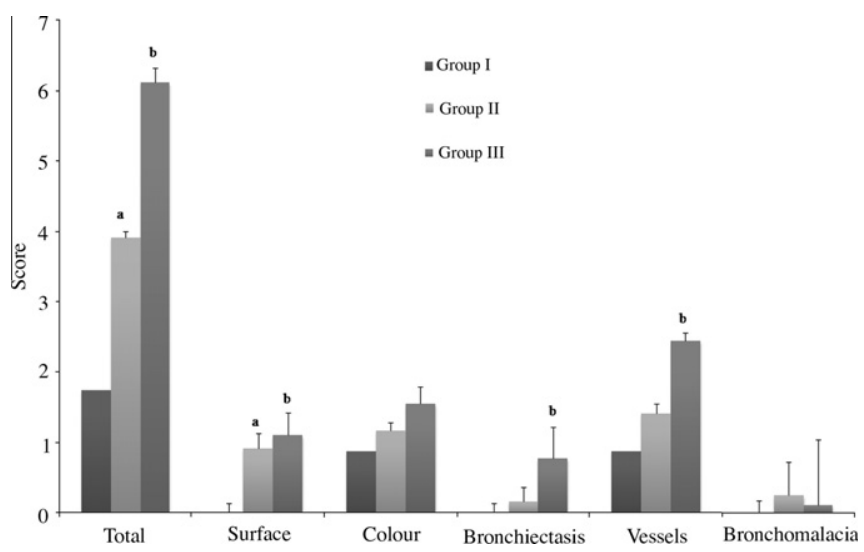

Fig. 1. Bronchoscopic scores for dogs in the three age groups. (a) Significant difference between group I and group II. (b) Significant difference between group I and group III.

\section{Correlation between BALF, radiographic and bronchoscopic scores}

There were no significant correlations between TCC and total radiographic or bronchial scores.

\section{Discussion}

The aim of this study was to determine whether there were significant age-related changes in bronchoscopic findings and BALF in the lower respiratory tract of the dog. The finding of such changes would have implications for the interpretation of data from studies of spontaneous canine respiratory disease or from experimental studies of induced respiratory disease in canine models.

Health status was based on physical examination, haematology, serum biochemistry, thoracic radiography and BALF analysis. Limited testing for viral or bacterial pathogens was undertaken and no serological testing for canine respiratory viruses was performed.

There were no significant differences in the total and differential blood cell counts in young and old dogs in this study. Dogs $<1$ year of age may have a relative lymphocytosis (Dunn, 2000; Toman et al., 2002; Comazzi et al., 2004), but the mean age of the young dogs in the present study was 2.1 years.

The total radiographic scores in the three age groups were not significantly different. This was surprising, since age-related differences have been reported in dogs, e.g. pleural thickening, increased interstitial and bronchial patterns, bronchial calcification and, rarely, hyperlucency in some parenchymal areas (Reif and Rhodes, 1966). These changes are correlated with histopathological alterations with age, including pleural and interstitial fibrosis, calcification of bronchial cartilage, focal emphysema and heterotopic bone formation (Reif and Rhodes, 1966).

The use of radiographic scoring systems is considered to be of questionable value, since the process of assigning scores is highly subjective (i.e. evaluator-dependent), healthy animals never have a zero radiographic score and a progressive increase in score can occur over time in healthy subjects (Mantis et al., 1998). These observations suggest that radiography is not the most accurate means of making a specific diagnosis of the nature of respiratory disease.

Total bronchoscopic scores were significantly higher in older dogs than in middle-aged and younger dogs. Three major differences were identified in older dogs: the presence of an irregular bronchial mucosa; the presence of bronchiectasis and visualisation of large vessels. We hypothesised that irregularity of the bronchial a

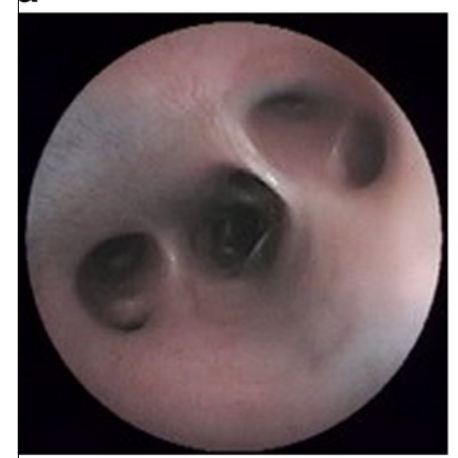

d

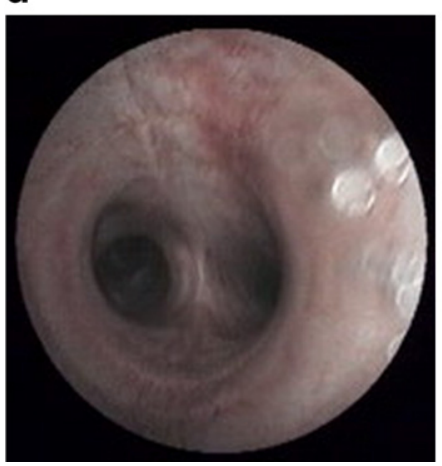

b

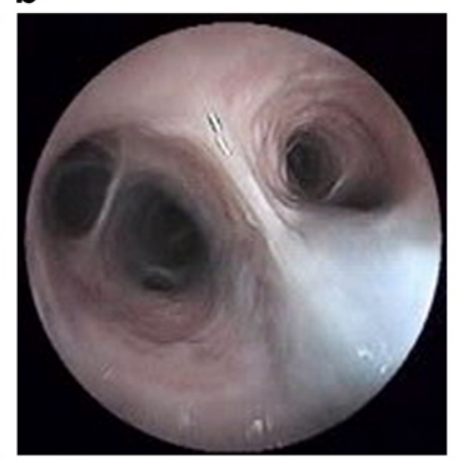

e

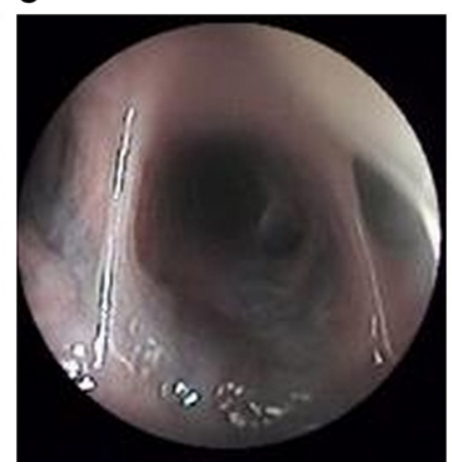

c

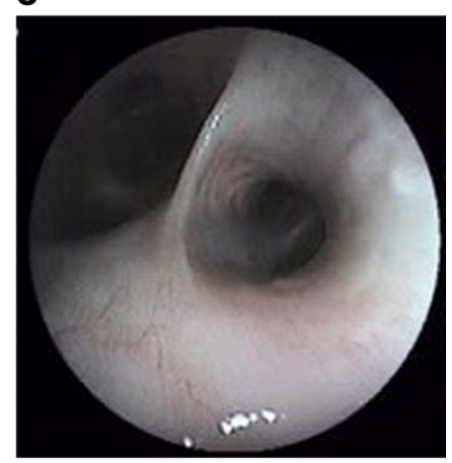

f

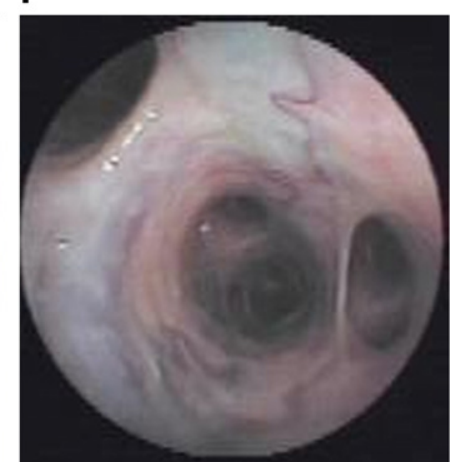

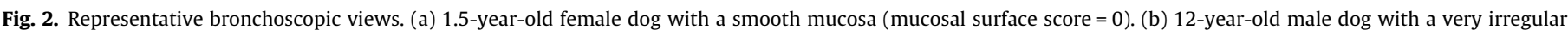

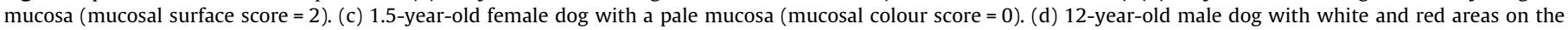
mucosa (mucosal colour score $=2$ ). (e) and (f) 12-year-old male dog with presence of large and protruding vessels (vessel score $=3$ ). 
mucosa may be related to the presence of subclinical chronic inflammatory changes. In a preliminary attempt to test this hypothesis, mucosal perendoscopic biopsies were obtained from a separate group of older dogs; however no inflammation was noted in these samples (data not shown).

It was hypothesised that the observed bronchiectasis in older dogs may be due to bronchial wall remodelling and loss of mucosal elasticity. Few previous studies have considered age-related changes in the components of the bronchial wall of the dog. Wheeldon and Pirie, (1974) applied a point counting method described in humans to quantify such components. In our study, no significant differences in the proportion of bronchial wall occupied by mucous glands or smooth muscle were found between young and old dogs (data not shown). Reif and Rhodes (1966) studied morphological and histopathological alterations in the lung of old dogs and found foci of interstitial fibrosis, emphysema, pleural fibrosis and bronchial cartilage calcification.

The presence of large, protruding and pulsating vessels was also a distinctive finding on bronchoscopic evaluation of older dogs in the present study. We hypothesise that this change might be related to bronchial wall alterations, such as thinning of the mucosa and/or vascular remodelling. Future investigations using high-resolution computed tomography, together with the use of threedimensional reconstruction techniques and contrast enhancement, might enable a more definitive assessment of airway mucosal and vascular changes, as described in humans (Hansell and Kerr, 1991; Corcoran et al., 1992; Schwickert et al., 1994; Song et al., 1998).

In the present study, younger dogs had a higher percentage of neutrophils in BALF compared with middle-aged and old dogs and a higher percentage of lymphocytes in BALF compared with middle-aged dogs. Children have higher percentages of lymphocytes in BALF than adult humans, which may reflect infection with respiratory viruses (Ratjen et al., 1994). Acute respiratory viral infections also occur with high frequency in young dogs, especially those that are unvaccinated (Buonavoglia and Martella, 2007). The dogs in the present study were all vaccinated and were free of clinical respiratory tract disease in the 3 month period preceding the study.

There was no statistically significant correlation between BALF TCC and the total radiographic and bronchial scores in the dogs in this study. Kirschvink et al. (2007) demonstrated an association between radiographic and bronchoscopic changes and an increased TCC in BALF in cats, while there were relatively poor correlations among radiography, bronchoscopy and BALF in other studies in dogs and cats (Norris et al., 2001, 2002). However, the major limitation of the present study is the small size of each group, which could partly explain the lack of significant differences.

\section{Conclusions}

Older dogs have a more irregular bronchial mucosa than younger dogs, with prominent vessels on bronchoscopic examination. In contrast, younger dogs have significantly more neutrophils within BALF than middle-aged and old dogs and more lymphocytes than middle-aged dogs. Age-related differences should be considered when interpreting bronchoscopic findings and BALF analysis in dogs with respiratory disease.

\section{Conflict of interest statement}

None of the authors of this paper has a financial or personal relationship with other people or organisations that could inappropriately influence or bias the content of the paper.

\section{Acknowledgements}

The authors thank Mrs. Sheila Jones and Mr. François Delvaux for their technical assistance.

\section{References}

Buonavoglia, C., Martella, V., 2007. Canine respiratory viruses. Veterinary Research 38, 355-373.

Clercx, C., Peeters, D., Snaps, F., Hansen, P., McEntee, K., Detilleux, J., Henroteaux, M., Day, M.J., 2000. Eosinophilic bronchopneumopathy in dogs. Journal of Veterinary Internal Medicine 14, 282-291.

Comazzi, S., Pieralisi, C., Bertazzolo, W., 2004. Haematological and biochemical abnormalities in canine blood: frequency and associations in 1022 samples. Journal of Small Animal Practice 45, 343-349.

Corcoran, H.L., Renner, W.R., Milstein, M.J., 1992. Review of high-resolution CT of the lung. Radiographics 12, 917-939.

Dunn, J., 2000. Disorders of leucocyte number. In: Day, M., Mackin, A., Littlewood, J. (Eds.), Manual of Canine and Feline Haematology and Transfusion Medicine. BSAVA, England, pp. 93-104.

Foster, S.F., Allan, G.S., Martin, P., Robertson, I.D., Malik, R., 2004. Twenty-five cases of feline bronchial disease (1995-2000). Journal of Feline Medicine and Surgery 6, 181-188.

Kirschvink, N., Kersnak, E., Leemans, J., Delvaux, F., Clercx, C., Snaps, F., Gustin, P., 2007. Effects of age and allergen-induced airway inflammation in cats: radiographic and cytologic correlation. The Veterinary Journal 174, 644-651.

Hansell, D.M., Kerr, I.H., 1991. The role of high resolution computed tomography in the diagnosis of interstitial lung disease. Thorax 46, 77-84.

Kuehn, N.F., Hess, R.S., 2004. Bronchoscopy. In: King, L.G. (Ed.), Textbook of Respiratory Disease in Dogs and Cats. Elsevier, USA, pp. 112-118.

Mantis, P., Lamb, C.R., Boswood, A., 1998. Assessment of the accuracy of thoracic radiography in the diagnosis of canine chronic bronchitis. Journal of Small Animal Practice 39, 518-520.

Mayer, P., Laber, G., Walzl, H., 1990. Bronchoalveolar lavage in dogs; analysis of proteins and respiratory cells. Zentralblatt für Veterinärmedizin. Reihe A. 37, 392-399.

McKiernan, B.C., 2003. Bronchoscopy in small animal practice. In: Proceedings of the Western Veterinary Conference, Las Vegas, Nevada.

Norris, C.R., Griffey, S.M., Samii, V.F., Christopher, M.M., Mellema, M.S., 2001. Comparison of results of thoracic radiography, cytologic evaluation of bronchoalveolar lavage fluid, and histologic evaluation of lung specimens in dogs with respiratory tract disease: 16 cases (1996-2000). Journal of the American Veterinary Medical Association 218, 1456-1461.

Norris, C.R., Griffey, S.M., Samii, V.F., Christopher, M.M., Mellema, M.S., 2002 Thoracic radiography, bronchoalveolar lavage cytopathology, and pulmonary parenchymal histopathology: a comparison of diagnostic results in 11 cats. Journal of the American Animal Hospital Association 38, 337-345.

Ratjen, F., Bredendiek, M., Brendel, M., Meltzer, J., Costabel, U., 1994. Differential cytology of bronchoalveolar lavage fluid in normal children. European Respiratory Journal 7, 1865-1870.

Reif, J.S., Rhodes, W.H., 1966. The lungs of aged dogs: a radiographic-morphologic correlation. Journal of the American Veterinary Radiology 7, 5-11.

Schwickert, H.C., Kauczor, H.U., Schweden, F., Schild, H.H., 1994. The anatomy of the bronchial arteries - their imaging with spiral CT. Röfo: Fortschritte auf dem Gebiete der Röntgenstrahlen und der Nuklearmedizin 160, 506-512.

Song, J.W., Im, J.G., Shim, Y.S., Park, J.H., Yeon, K.M., Han, M.C., 1998. Hypertrophied bronchial artery at thin-section CT in patients with bronchiectasis: correlation with CT angiographic findings. Radiology 208, 187-191.

Toman, M., Faldyna, M., Knotigova, P., Pokorova, D., Sinkora, J., 2002. Postnatal development of leukocyte subset composition and activity in dogs. Veterinary Immunology and Immunopathology 87, 321-326.

Whaley, S.L., Muggenburg, B.A., Seiler, F.A., Wolff, R.K., 1987. Effect of aging on tracheal mucociliary clearance in beagle dogs. Journal of Applied Physiology 62, 1331-1334.

Wheeldon, E.B., Pirie, H.M., 1974. Measurement of bronchial wall components in young dogs, adult normal dogs, and adult dogs with chronic bronchitis. American Review of Respiratory Disease 110, 609-615. 